\title{
Cationic polymer-in-salt electrolytes for fast metal ion conduction and solid-state battery applications
}

Fangfang Chen ( $\square$ chenf@deakin.edu.au )

Deakin University https://orcid.org/0000-0002-8004-1720

Xiaoen Wang

Deakin University https://orcid.org/0000-0001-7713-7062

Michel Armand

CIC energigune https://orcid.org/0000-0002-1303-9233

Maria Forsyth

Deakin University https://orcid.org/0000-0002-4273-8105

\section{Article}

Keywords: batteries, engery devices, polymer electrolytes

Posted Date: May 25th, 2021

DOl: https://doi.org/10.21203/rs.3.rs-532893/v1

License: (c) (i) This work is licensed under a Creative Commons Attribution 4.0 International License. Read Full License

Version of Record: A version of this preprint was published at Nature Materials on July 28th, 2022. See the published version at https://doi.org/10.1038/s41563-022-01319-w. 


\section{Abstract}

Polymer electrolytes provide a safe solution for all-solid-state high energy density batteries. Materials that meet the simultaneous requirement of high ionic conductivity and high transference number remain a challenge, in particular for new battery chemistries beyond Lithium such as $\mathrm{Na}, \mathrm{K}$ and $\mathrm{Mg}$. Herein, we demonstrate the versatility of a polymeric ionic liquid (PolyIL) as a solid-state solvent to achieve this goal for both $\mathrm{Na}$ and $\mathrm{K}$. Using molecular simulations, we predict and elucidate fast metal ion transport in PolylLs through a structural diffusion mechanism in a polymer-in-salt environment, facilitating a high transference number. Experimental validation of these computational designed $\mathrm{Na}$ and $\mathrm{K}$ polymer electrolytes gives high ionic conductivities of $1.010^{-3} \mathrm{~S} \mathrm{~cm}-1$ at $80^{\circ} \mathrm{C}$ and an exceptional $\mathrm{Na}^{+}$ transference number of $\sim 0.57$. Electrochemical cycling of a sodium anode also demonstrates an ultralow overpotential of $40 \mathrm{mV}$ and stable long term performance of more than 100 hours in a symmetric cell. PolylL-based polymer-in-salt strategies for novel solid-state electrolytes thus offer a promising route to design high performance next generation sustainable battery chemistries.

\section{Main Text}

High-energy-density energy storage technology requires the use of a new generation of anode materials, e.g. alkali metal anodes ( $\mathrm{Li}, \mathrm{Na}$ or K) with high theoretical capacity, and in the case of $\mathrm{Na}$ and $\mathrm{K}$, also having a higher abundance in the earth's crust. Such reactive anodes necessitate the discovery of compatible electrolyte materials to support the safe and long-term cycling of these new energy storage devices. Polymer electrolytes (PEs) are now accepted as an enabler of the ultimate solid-state highperformance batteries. ${ }^{1-4}$ Given the appropriate chemistry and materials properties, these materials can address the essential safety issue, i.e. prohibiting the formation of dendrites on highly reactive metal anodes through their optimal mechanical properties, in addition to formation of a potentially beneficial solid electrolyte interphase (SEI) layer, depending on their chemistry. ${ }^{5}$ Furthermore, PEs possess the additional advantages of high thermal stability, high electrochemical stability and ideal flexibility that help to improve battery safety and cycle life.

The earliest PEs for battery applications were proposed based on archetypal polyethylene oxide (PEO) or copolymers of PEO, ${ }^{6,7}$ The polar ether functional group in the polymer backbone coordinates $\mathrm{Li}^{+}$and drives its diffusion through the strongly coupled backbone motion. These PEO based systems have been normally restricted to using relatively low salt concentrations (e.g. $\left.\mathrm{EO}: \mathrm{Li}^{+}=20\right)$ in order to obtain maximum ionic conductivity, ${ }^{8}$ although the value is still below the minimum conductivity required for practical applications at moderate temperatures. They also suffer low $\mathrm{Li}^{+}$transference numbers $\left(T_{\mathrm{Li}}{ }^{+} \sim\right.$ 0.2 ) as the 'free' anions, such as bis(trifluoromethanesulfonyl)imide ( $\mathrm{TFSI}^{-}$), dominate the conductivity whereas the $\mathrm{Li}^{+}$motion is restricted by the strong EO-Li coordination. ${ }^{9-11}$ Although PEO based PEs have been commercialized by Bolloré group, their use is still limited, in particular to positive electrode material operating below $4 \mathrm{~V}$ vs $\mathrm{Li}^{+} / \mathrm{Li}^{\circ}$. A variety of polymer design strategies have been proposed to improve $\mathrm{PE}$ performance, e.g. designing new anion chemistries with restricted motions, ${ }^{12}$ using polycarbonate groups 
to enhance dissociation of lithium salt, ${ }^{11,13,14}$ using block copolymers and crosslinking of polymer chains to improve mechanical properties and ionic conductivities, etc.. ${ }^{15,16}$ Besides those, the search for new polymer materials with higher metal ion transference number and conductivity is ongoing.

One approach to maximise the transference number of the metal ions is to use polymers that have any other ions chemically tethered to backbones (also known as polyelectrolytes) to immobilize their motion. In the case of polyanionic materials, in which anions are chemically bonded to polymer backbones, cation conduction dominates and the cation transference number tends to unity. ${ }^{17}$ Thus in the case of a lithium salt polyelectrolyte, the ionic conductivity is completely derived from $\mathrm{Li}^{+}$conduction which is highly desirable in lithium batteries in order to overcome problems related with anion concentration gradients. Despite progress such single ion conductors with any plasticizers/solvents still typically suffer very low ionic conductivities that hinder their applications. ${ }^{18,19}$

Recently, polycationic PEs have emerged as potential solid-state solvents for lithium salts, in particular the monomer is a polymerizable IL cation, showing good performance in Lithium batteries. ${ }^{20,21}$ This material is termed a polymeric or polymerized ionic liquid (PolylL), ${ }^{22}$ which inherits both the excellent electrochemical performance of ILs and good physical, thermal and mechanical properties of polymers. ${ }^{23,} 24$ Interestingly, these materials have been shown to support the dissolution of extremely high lithium salt concentrations, such that the ratio of $\mathrm{Li}^{+}$: polycation is greater than 1 , without sacrificing ionic conductivity, ${ }^{21,25,26}$ due to a unique Li-anion-polycation co-coordination structure ( $\mathrm{Li}^{+}$ions are distributed around the polycation backbone by anion-bridging $\left.{ }^{26}\right)$. This abnormal concentration conductivity positive correlation differentiates PolyILs significantly from traditional PEs such as PEObased electrolytes, ${ }^{27}$ but is in line with the 'polymer-in-salt' electrolytes (PISE) ${ }^{28-30}$ proposed by C. Austen Angell decades ago. In such cases, ion mobility will begin to increase again while the glass transition temperature $\left(T_{\mathrm{g}}\right)$ decreases, once the salt is the dominant component in the PISE system. ${ }^{28}$ However, the PolylL is unique in that this salt concentration-conductivity positive correlation is also applicable to the low salt concentration range. ${ }^{21,26}$

The fast $\mathrm{Li}^{+}$conduction in "polylL-in-salt" should be closely related to its transport mechanism. A recent report using computational methods, suggested a vehicular $\mathrm{Li}^{+}$diffusion mechanism in PolylL electrolytes which, however, only focused on a low to medium salt concentration range (i.e. $\mathrm{Li}^{+}$: cation repeat unit $£ 0.4) .{ }^{31}$ It is likely that this mechanism is concentration dependent, as has been noted for liquid electrolytes, since the salt concentration will alter the $\mathrm{Li}^{+}$coordination environment. ${ }^{32-36}$ Given the recent promising demonstration of polylL-in-salt systems in Li metal batteries, understanding the transport mechanisms in these new PISE materials could open up the possibility of designing PEs with high conductivity and high target ion transference number. Furthermore, while several systems have been proposed for Li devices, materials focusing on other battery chemistries such as $\mathrm{Na}$ and $\mathrm{K}$ are sparse and would enable solid-state batteries based on these more abundant materials. 
Thus, in this work we used a computational approach to investigate the transport of $\mathrm{Na}^{+}$and $\mathrm{K}^{+}$in PolylL based PISEs and discovered a rapid diffusion of alkali metal ions through a structural diffusion mechanism. Under the premise of weak interaction between metal ions and anions, the salt-concentrated environment favors fast metal ion diffusion. We also investigated the solvation and diffusion of the multivalent $\mathrm{Mg}^{2+}$ in these PolylLs and discussed the challenges in achieving high $\mathrm{Mg}^{2+}$ conduction in such systems. Finally, as a proof of concept, we validated the computational findings by experimental measurement of conductivity as well as in application of a Na based PolylL PISE in a sodium symmetric cell, which showed excellent stable cycling behavior at a capacity of $0.5 \mathrm{mAh} \mathrm{cm} \mathrm{m}^{-2}$ per cycle for over 100 cycles.

\section{Computational design optimal polylL-in-salt systems}

The PolyIL studied in this work is poly(diallyldimethylammonium)bis(fluorosulfonyl)imide (PDADMA FSI) (Supplementary Fig1e). Our previous experimental work has studied the use of this polymer with increasing amounts of LiFSI salt at polycation to $\mathrm{Li}^{+}$mole ratios from 2:1 to 1:6. Careful experimental measurements were used to find the optimum conductivity. ${ }^{26}$ However, such an approach to electrolyte design is both time consuming and costly and here we sought a more efficient computational approach. Based on MD simulations in that earlier work we learned that the optimal salt concentration $(1: 1.5)$ determined in PDADMA FSI/Li FSI allows the most lithium ions to be homogeneously distributed around polycationic backbones by co-coordinating with anions. At higher $\mathrm{Li}^{+}$ratio, excessive $\mathrm{Li}^{+}$form molten salt-like aggregates. Although in silico, these previous simulations suggested that $\mathrm{Li}^{+}$diffusion could be further enhanced with these aggregates, this could not be realised in our experiments as those $\mathrm{Li}^{+}$-rich domains eventually transform into the less conductive crystalline salt-like new phase. Nevertheless, based on these understandings, we hypothesize here that a close-to-optimal salt concentration could at least be identified from a PolyIL PISE composition that gives the 'saturated' metal ion distribution around polymer chains, and can be determined via quantifying three different anion coordination states: (1) the polycation-anion single-coordination state $\left(\mathrm{P}^{+}-\mathrm{A}^{-}, \mathrm{Fig} 1 \mathrm{a}\right)$ that can be seen in the case of neat polylL or with low salt concentrations; (2) the metal ion-anion single-coordination state $\left(\mathrm{Me}^{+}-\mathrm{A}^{-}, \mathrm{Fig} 1 \mathrm{c}\right)$ as seen in the molten salt-like regime; and (3) the polycation-anion-metal ion co-coordination state $\left(\mathrm{P}^{+}-\mathrm{A}^{-}-\mathrm{Me}^{+}, \mathrm{Fig}\right.$ 1b). We use this to investigate alternative salt chemistries including $\mathrm{NaFSI}, \mathrm{KFSI}$ and $\mathrm{Mg}[\mathrm{FSI}]_{2}$.

The PolyIL/NaFSI systems at two $\left(\mathrm{P}^{+}: \mathrm{Na}^{+}\right)$ratios of $1: 2$ and $1: 4$ (termed $\mathrm{Na} 12$ and $\mathrm{Na} 14$ ) were firstly examined. The 1:2 cation ratio was chosen for the NaFSI system as it is close to the optimal 1:1.5 ratio of the lithium system, and $\mathrm{Na}^{+}$requires to be coordinated with slightly higher number of $\mathrm{FSI}^{-}$than $\mathrm{Li}^{+}$. The 1:4 cation ratio as a higher salt concentration was also examined. The snapshots in Fig $1 \mathrm{~d}-1 \mathrm{e}$ show the selected structure of the equilibrated $\mathrm{Na} 12$ and $\mathrm{Na} 14$ systems, including $\mathrm{FSI}^{-}$only in two singlecoordination states (purple for $\mathrm{P}^{+}-\mathrm{FSI}^{-}$and green for $\mathrm{Na}^{+}-\mathrm{FSI}^{-}$), the cationic polymer matrix without $\mathrm{H}$ atoms (aqua blue) and the $\mathrm{Na}^{+}$ions (yellow). The lowest percentage of $\mathrm{P}^{+}-\mathrm{FSI}^{-}$or $\mathrm{Na}^{+}-\mathrm{FSl}^{-}$coordination occurs in the Na12 system, with the highest state being the co-coordination state consisting of $97 \% \mathrm{FSI}^{-}$, 
which is even higher than that (92\%) in the optimal PolyIL/LiFSI (1: 1.5) system. This high percentage of co-coordination suggests that the 1:2 ratio can be selected as the initial concentration for the $\mathrm{Na}^{+}$system. For the $\mathrm{Na} 14$ case, with the added salt doubled, the percentage of $\mathrm{FSI}^{-}$in the $\mathrm{Na}^{+}-\mathrm{FSI}^{-}$coordination state increases significantly to $19.7 \%$, indicating the growth of the $\mathrm{Na}^{+}-\mathrm{FSI}^{-}$rich domains at higher $\mathrm{Na}^{+}$ concentration, and this change is consistent with the LiFSI case ${ }^{26}$.

The 1:2 cation ratio was examined next for both $\mathrm{K}^{+}$and divalent $\mathrm{Mg}^{2+}$ systems to investigate anion states and the possible optimal salt concentrations. As shown in Fig $1 \mathrm{f}$ and the snapshots in Supplementary Fig 1, there is an obvious increase in the percentage of $\mathrm{FSI}^{-}$in $\mathrm{FSl}^{-}-\mathrm{Me}^{+}$coordination state with $\mathrm{Na}^{+}<\mathrm{K}^{+}<\mathrm{Mg}^{2+}(2.2 \%, 8 \%$ and $23.1 \%$ respectively), while the co-coordination state decreases to $91 \%$ and $73.9 \%$ for $\mathrm{K} 12$ and $\mathrm{Mg} 12$, respectively. This suggests us to test a lower salt concentration, with the expectation of increasing the co-coordination state of $\mathrm{FSI}^{-}$as confirmed in Fig $1 \mathrm{f}$. We found that, at 1:1 ratio, the co-coordination state increases to $94 \%$ and $85.4 \%$ in the $\mathrm{K} 11$ and $\mathrm{Mg} 11$ systems, respectively. In the $\mathrm{K} 11$, the percentage of $\mathrm{FSI}^{-}$in both single-coordination states is relatively low, while in the Mg11, the percentage of both is $7.3 \%$, which may be due to the difficulty in completely dissociating the Mg salt.

Interestingly, when comparing cation ratios that can obtain nearing the maximum co-coordination anion state, i.e. 1:1.5 for $\mathrm{Li}^{+}, 1: 2$ for $\mathrm{Na}^{+}$and 1:1 for $\mathrm{K}^{+}$, they are ordered differently to alkali metals in the periodic table $(\mathrm{Li}<\mathrm{Na}<\mathrm{K})$. This variation in cation ratio could be related to the difference in cation-anion coordination abilities. For example, for a given 1:2 cation ratio, the coordination number (CN)

(Supplementary table 1) of $\mathrm{P}^{+}$- $\mathrm{FSI}^{-}$in the $\mathrm{Na} 12$ (8.7) is greater than that in the $\mathrm{K} 12$ (8.1), but an opposite situation is observed for the $\mathrm{CN}$ of $\mathrm{Me}^{+}-\mathrm{FSI}^{-}$coordination which is greater in the $\mathrm{K} 12$ (6.4) than in the $\mathrm{Na} 12$ (5.6). Therefore, the number of metal ions in the co-coordination state bridged by $\mathrm{FSI}^{-}$should also be less in the $\mathrm{K} 12$ and more in the Na12 system. The $\mathrm{CN}$ also helps to understand the extensive $\mathrm{Mg}-\mathrm{FSI}$ enriched domains in the Mg12 system. Since the amount of $\mathrm{FSI}^{-}$is doubled whereas the $\mathrm{CN}$ of both $\mathrm{Mg}^{2+}-\mathrm{FSI}^{-}$(5.7) and $\mathrm{P}^{+}-\mathrm{FSI}^{-}$(9.2) are not, this results in excessive $\mathrm{FSI}^{-}$anions that mainly coordinate with $\mathrm{Mg}^{2+}$. A more detailed structural analysis of the cation-anion coordination by radial distribution function (RDF, supplementary Fig 2) can be found in the supplementary information.

\section{The impact of chemical environmental on ion diffusion}

In this section, the diffusion of ions is investigated to evaluate the ionic conductivity in sodium, potassium and magnesium electrolytes. The ion diffusion is compared among three electrolytes of K12, $\mathrm{Na} 12$ and Mg11 having the same concentration of anions via the mean square displacement (MSD) of metal ions and anions (Supplementary Fig 3). In general, ion diffusion in the alkali metal ion systems is 1-2 orders of magnitude higher than that in the magnesium system. At a temperature of $353 \mathrm{~K}$, the diffusion of metal ions and anions in the K12 system is faster than that in the Na12 system. Similar results were also reported in an ionic liquid electrolyte $N$-methyl- $N$-propylpyrrolidinium bis(fluorosulfonyl)imide ( $\left.\mathrm{C}_{3} \mathrm{mpyrFSI}\right)$. When mixed with the same amount of KFSI or NaFSI salt, the IL 
system with the KFSI salt has higher ionic conductivity. ${ }^{37}$ This may be due to the lower Lewis acidity of potassium, making its interaction with the anion weaker than sodium, which is confirmed by the density functional theory (DFT) calculation of the binding energy $\left(E_{\mathrm{b}}\right)$ between metal ions and anions.

The relative diffusivities of metal ions and anions in monovalent alkali metal salts are different from those in divalent magnesium salt. The diffusivity of alkali metal metal ions is higher than that of anions. Taking into account that polycations are immobile, this indicates that a higher alkali metal ion transference number can be obtained, which is in sharp contrast with traditional PEO-based electrolyte systems. This was confirmed in the case of $\mathrm{Na}$ via experimental validation in next section. On the other hand, the opposite behaviour is observed for the $\mathrm{Mg}^{2+}$ system, which shows a lower $\mathrm{Mg}^{2+}$ diffusivity relative to $\mathrm{FSI}^{-}$in addition to being the least diffusing cations amongst all simulated systems. Next, we conducted a detailed structural analysis of the targeted ions having distinct fast and slow diffusion, and studied the environmental factors that affect the fast ion diffusion. The Na12 and Mg11 systems were selected for this analysis.

Firstly, the mode of interaction between the arbitrary selected fast and slow diffusive ions (defined in supplementary Fig 4) was studied by calculating the RDF between the two selected groups (i.e. fast vs. fast or fast vs. slow group) as presented in Figure 2. Considering firstly the correlation between $\mathrm{FSI}^{-}$and $\mathrm{Na}^{+}$diffusion, Fig $2 \mathrm{c}$ and $2 \mathrm{~d}$ show that fast $\mathrm{FSI}^{-}$tends to coordinate with fast $\mathrm{Na}^{+}$, and similarly, slow $\mathrm{FSI}^{-}$tends to coordinate with slow $\mathrm{Na}^{+}$, which is inferred from their more prominent RDF peaks and higher $\mathrm{CN}$ in comparison to the coordination between the fast and slow ions. This also indicates that the movement of $\mathrm{Na}^{+}$and $\mathrm{FSI}^{-}$has a strong correlation. Secondly, Fig $2 e$ and $2 f$ show the RDF of polycation $-\mathrm{FSI}^{-}$and polycation $-\mathrm{Na}^{+}$, respectively, revealing that the fast $\mathrm{FSI}^{-}\left(\right.$or $\mathrm{Na}^{+}$) has less coordination with polycations, which is in contrast to the slow $\mathrm{FSI}^{-}\left(\mathrm{or} \mathrm{Na}^{+}\right)$. This suggests that the diffusion of ions is slower near polycations or when coordinating with more polycations. Finally, Fig $2 \mathrm{~g}$ and $2 \mathrm{~h}$ investigate the number of $\mathrm{Na}^{+}$and $\mathrm{FSI}^{-}$ions surrounding the selected fast and slow $\mathrm{Na}^{+}$. Interestingly, there are always more $\mathrm{Na}^{+}$and $\mathrm{FSI}^{-}$ions surrounding the fast $\mathrm{Na}^{+}$, i.e. the environment rich in $\mathrm{Na}^{+}$and $\mathrm{FSI}^{-}$facilitates the faster metal cation dynamics, as demonstrated by a snapshot in Fig $2 \mathrm{~b}$. The fast $\mathrm{Na}^{+}$(green) has the molten Na-FSI salt-like environment which is commonly seen in superconcentrated ionic liquid electrolytes. ${ }^{32,36,38}$ In contrast, the environment of slow $\mathrm{Na}^{+}$(blue) contains fewer $\mathrm{FSI}^{-}$and $\mathrm{Na}^{+}$within the same distance range. This result suggests that the evolution of molten salt regions at high salt concentrations can promote metal ion diffusion in the polylL, thus providing a theoretical guideline for future polymer electrolyte design, i.e. by enhancing the molten salt like domains in a polylL matrix. We experimentally validate this concept later in the paper.

In the case of the Mg11 system we observe some contrasting behaviour. The fast $\mathrm{FSI}^{-}$preferentially coordinates with the polycationic backbone (Supplementary Fig 5a - 5b). Furthermore, the environment of the fast $\mathrm{Mg}^{2+}$ is also dominated by more polycations, with a lower $\mathrm{CN}$ for both $\mathrm{FSl}^{-}$and $\mathrm{Mg}^{2+}$ (Supplementary Fig 5d-5f). In other words, the faster $\mathrm{Mg}^{2+}$ and $\mathrm{FSI}^{-}$are not found in domains rich in 
$\mathrm{Mg}^{2+}$ and $\mathrm{FSl}^{-}$ions, which is the opposite situation to that shown in the alkali metal salt systems. We can understand this by considering the relative binding interaction between the metal ions and $\mathrm{FSI}^{-}$(Fig 2b); for $\mathrm{Mg}^{2+}$ this is so strong that it dramatically reduces the diffusion of both ions. Therefore, in these high salt content PolyIL electrolytes, the ionic conductivity enhancement requires the metal ion-anion interaction to be weak enough to allow fast exchange in the coordination environment, which we discuss in more detail below. For the development of polylL electrolytes that conduct multivalent ions (e.g. $\mathrm{Mg}^{2+}$, $\mathrm{Zn}^{2+}$ etc.), designing metal ion-anion interaction of similar magnitude to the alkali metal ion -FSI binding energy is the key.

\section{Metal ion diffusion mechanisms}

In this section, we show that the dominant metal ion diffusion mechanism in the polylL-in-salt system, is a structural diffusion mechanism as opposed to the vehicular diffusion mechanism suggested in the low salt concentration polylL ${ }^{31}$, or the diffusion through polymer backbone motion in the traditional PEs (e.g. PEO). If we consider the nearest coordination structure of a given metal cation to be its 'cage' we can then quantitatively determine the cage reorganisation dynamics and their relationship to ion diffusion processes. Three types of metal ion diffusion mechanisms can be distinguished; vehicular, structural and hopping. We define the period from the initial formation of an old cage $A$ to the formation of a new cage $B$ as the 'cage-restructuration period' (Fig 3a). This period will be longer for the vehicular mechanism since the metal ions will diffuse with the cage. In contrast, in the case of the structural mechanism, this time period will be much shorter since the diffusion of ions occurs through the cage restructuring. The cage restructuration in the hopping mechanism occurs instantaneously and causes ion migration via an ion hop from one cage environment to another.

In Fig 3b The ion cage restructuring in Na12 and K12 systems is analysed through the cagerestructuration correlation function $C(t)$ (with a specific definition described in the supplementary file). The decay in this function is caused by the occurance of cage restructuring, i.e. new anions participate in metal ion's coordination shell. $C(t)$ decays to 0.9 almost immediately for both $\mathrm{K} 12$ and $\mathrm{Na} 12$ systems. It takes nearly $3.5 \mathrm{~ns}$ to decay to close to 0.01 in the $\mathrm{K} 12$, which is significantly faster than the $12.7 \mathrm{~ns}$ required in Na12 system for most metal ions to complete one cage restructuration process.

Fig $3 \mathrm{c}$ and $3 e$ analyse the frequency of different cage-restructuration periods in the $30 \mathrm{~ns}$ trajectory, and accumulates the deviation distances of metal ions in all the same cage-restructuration periods. The average deviation distance of each restructuration period is given in Fig $3 \mathrm{~d}$. The accumulative distance profile and the frequency profile present a similar pattern, indicating that the distance the metal ion travels is positively correlated to the frequency of the cage-restructuration period, but has no relationship with the cage-restructuration time, as suggested by Fig $3 \mathrm{~d}$. This further supports the structural diffusion mechanism for metal ions, and demonstrates that the key to improve the metal ion conduction is to accelerate the reorganization of their coordination shells. 
Finally, the frequency of an instant hopping event was identified in order to evaluate the hopping diffusion mechanism. This is when the cage restructuration is completed between two consecutive frames in the MD trajectory. Only 7 events were detected in more than 2 million inspections, indicating that his mechanism would only rarely contribute to diffusion, and thereby ionic conductivity, in these electrolytes.

\section{Experimental validation of in-silico predictions}

Based on simulation results, we conducted experimental validations to verify the composition dependence and high ion conductivity in these PolyIL PISE electrolytes. The Na11, Na12, Na14, K11 and K12 electrolytes were prepared and their thermal properties, ionic conductivities and electrochemical behaviour were studied. Fig 4 a presents the $T_{\mathrm{g}}$ of those systems with that of the Li11 and Li12 systems from our previous study. ${ }^{26} T_{\mathrm{g}}$ is normally used as an indicator of ion dynamics for those traditional electrolytes whose ion dynamics are highly correlated with the relaxation process of the polymer backbones. For all polylL systems studied here in Fig $4 \mathrm{a}, T_{\mathrm{g}}$ decreases with the increase of metal ion concentration, for example, $T_{g}$ follows $\mathrm{Na} 11>\mathrm{Na} 12>\mathrm{Na} 14$. The decrease in $T_{g}$ is due to the increase in metal ion-anion-polycation co-coordination so that the polycation-anion interaction and the ionic crosslinking of polymer chains is reduced, thereby increasing the local dynamics that controls $T_{\mathrm{g}}$. Excitingly, the lowest $T_{\mathrm{g}}$ of $\mathrm{Na} 14$ could support the modelling prediction regarding the positive role of the molten salt-like regions on ion dynamics in such systems.

The conductivity was measured for $\mathrm{Na} \mathrm{11,} \mathrm{Na} 12, \mathrm{~K} 11$, and K12 systems in Fig 4b. Na12 has higher conductivities than $\mathrm{Na} 11$ over the whole temperature range, and $\mathrm{s}_{\mathrm{Na} 12}$ reaches a value around $1.0 \mathrm{mS} \mathrm{cm}^{-}$ 1 at $80^{\circ} \mathrm{C}$, indicative of fast ion dynamics in this electrolyte. The conductivities of $\mathrm{K} 11$ and $\mathrm{K} 12$ systems measured during the $1^{\text {st }}$ heating cycle, however, do not reflect their difference in $T_{\mathrm{g}}$. The conductivity of $\mathrm{K} 11$ is higher than that of $\mathrm{K} 12$ except at a temperature of $100^{\circ} \mathrm{C}$, although the latter has a lower $T_{\mathrm{g}}$. Both are also less conductive than their sodium counterparts, which is also contrary to MD predictions. The DSC measurements on the two K-based systems (Supplementary, Fig 6a) suggest that this lower conductivity is likely due to the presence of a second crystalline phase, as we previously noted for $\mathrm{Li}$ systems. Encouragingly, a dramatic increase in the conductivity of K12 is seen during the $2^{\text {nd }}$ heating cycle, when the second crystalline phase is completely eliminated, as indicated by the disappearance of the melting peak in DSC (Supplementary Fig $6 \mathrm{~b}$ ). The conductivity of $\mathrm{K} 12$ is then significantly higher than $\mathrm{K} 11$ above $60^{\circ} \mathrm{C}$ and also surpasses $\mathrm{Na} 12$ above $80^{\circ} \mathrm{C}$, and this result is consistent with our MD predictions at $353 \mathrm{~K}$. It also confirms the conclusion that maintaining a single, amorphous polymer phase is essential to ensure high ionic conductivity in such high salt concentration PEs. It should also be noted that despite all PolylLs having the same FSI anion, their measured $T_{\mathrm{g}}$ is not completely correlated with the ionic conductivity across PolylLs with different alkali metal salts. For example, although the Li12 has the lowest $T_{\mathrm{g}}$ in Fig $4 \mathrm{a}$, its ionic conductivity is around $0.6^{\prime} 10^{-4} \mathrm{~S} \mathrm{~cm}^{-1}$ at $80^{\circ} \mathrm{C}$, which is lower than that of $\mathrm{Na} 12\left(1.02^{\prime} 10^{-3} \mathrm{~S} \mathrm{~cm}^{-1}\right)$ or $\mathrm{K} 12\left(1.2^{\prime} 10^{-3} \mathrm{~S} \mathrm{~cm}^{-1}\right)$ (compared at $2^{\text {nd }}$ heating) . 
Fig 4c shows the chronoamperometry profile of a symmetric cell assembled with the Na12 electrolyte and the Nyquist plots before and after DC polarization (inset). Although there is a fast decay of current in the first hour, the current is still maintained at a high level, indicative of relatively high $\mathrm{Na}^{+}$current (initial current $0.054 \mathrm{~mA}$ vs steady state current $0.044 \mathrm{~mA}$ ). Further analysis via the Bruce-Vincent method confirms an $\mathrm{Na}^{+}$transference number of 0.57 at $80^{\circ} \mathrm{C}$, which is significantly higher than that of PEONaFSI system $\left(t_{\mathrm{Na}+}=0.16\right)$ as previously reported by Zhou et al. ${ }^{39}$

The Na plating/stripping behaviours were also evaluated by symmetric Na cell cycling tests. As shown in Fig 4d, the Na12 electrolyte can sustain long term, stable Na plating and stripping under a high current

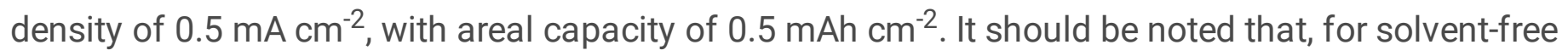
electrolytes, the current density applied in this case is much higher than that of state-of-the-art electrolyte systems. For example, a current density of $0.1 \mathrm{~mA} \mathrm{~cm}^{-2}$ was used for PEO/NaFSI electrolytes by Hu et al. ${ }^{40}$ More surprisingly, the polarization voltage for the Na12 electrolyte is relatively low, around $100 \mathrm{mV}$ (inset in Figure 5b), even at such a high current density. We ascribe this superior and stable plating/stripping performance to a high ionic conductivity and high transference number in the PISE. The preliminary test on the Na14 system (Supplementary Fig 7) also show very promising results with the higher steady state $\mathrm{Na}^{+}$current at $0.064 \mathrm{~mA}$ (initial current $0.084 \mathrm{~mA}$ ) and higher $\mathrm{Na}$ transference number of 0.63 obtained at $80^{\circ} \mathrm{C}$, and overpotential when cycling at high current densities. However, in this case the molten salt regime is metastable and transforms through crystallization after a few days, and thus future work will focus on stabilizing such systems.

\section{Conclusions}

Through computational design and detailed investigation of coordination structures and ion dynamics in polylL-in-salt based solid polymer electrolytes, we demonstrate that such systems have the versatility for the design of solid polymer electrolytes for multiple battery chemistries, including Li, Na and K, having high metal ion transference numbers and high conductivities. The optimal compositions can be more efficiently predicted based on simulation through analysis of the co-coordination state of anions in the polycationic polylL, with the maximum percentage of anion in this state being a minimum requirement. Specifically, the simulations suggest that metal ions diffuse in the polylL-in-salt system mainly through a structural diffusion mechanism and a rapid restructuring of the metal ion coordination environment is critical to achieving high metal ion diffusivity. The evolution of a molten salt-like region within the polylL facilitates rapid metal ion diffusion provided that the binding energy of salt is not excessive. We have validated these computational predictions through experimental measurement of ionic conductivity and transference number and demonstrated the outstanding electrochemical performance of the Na12 system under symmetric cell cycling, thereby demonstrating the significant potential of such polymer systems in the development of future all-solid-state high energy density batteries. This work opens up a cost effective computational approach to polymer electrolyte design based on Poly ILs with screening different anion/ cation chemistry as well as different metal salt chemistry and composition. 


\section{Methods}

\section{Molecular Simulations}

The molecular dynamics simulation was conducted using DL_Poly Classic software. ${ }^{41}$ The PDADMA FSI consists of 12 PDADMA chains with each having 12 repeat cationic units, and $144 \mathrm{FSI}$ anions. The number of salt ( $\mathrm{NaFSI}, \mathrm{KFSI}$ and $\left.(\mathrm{MgFSI})_{2}\right)$ is decided according to different (Polycation : metal ion) ratios of 1:1, 1:2 or 1:4 (given in Supplementary, Table 2). All polymers and ions were randomly placed in a cubic box using Packmol. ${ }^{42}$ The initial structures are equilibrated at $600 \mathrm{~K}$ and then cooled down to 393 $\mathrm{K}$ and then $353 \mathrm{~K}$. At each temperature, the system is equilibrated for $2 \mathrm{~ns}$ in a NPT ensemble, using Berendsen thermostat and barostat. The system is equilibrated again for $1 \mathrm{~ns}$ at $353 \mathrm{~K}$ using a NóseHoover thermostat and a Hoover barostat with relaxation constants of 0.5 and 5.0 ps each, and the extra $1 \mathrm{~ns}$ production run is followed for structure analysis. The velocity-verlet algorithm is adopted for integration. The time step is $1 \mathrm{fs}$ and the pressure is $1 \mathrm{~atm}$. The cutoffs for van der Waals force and the real space of Ewald were $12 \AA$. The Ewald summation method with a precision of $1^{\prime} 10^{-6}$ is used to treat Coulomb interaction in a periodic system. All C-H bonds are constrained. A subsequent production run for dynamics analysis is conducted in a NVE ensemble for $30 \mathrm{~ns}$ using a time step of $2.0 \mathrm{fs}$. The equilibrium state is checked through either total energy and volume for NPT calculation or total temperature and pressure for $N V E$ calculation, which should not increase or decrease throughout the production run. The force field parameters of the $\mathrm{FSI}^{-}$and metal ions aree taken from either CL\&P force field (2018 version). ${ }^{43}$ Those for the PDADMA are generated using the online OPLS_AA force field generator ${ }^{44}$ and validated in our previous work. ${ }^{26} \mathrm{~A}$ uniform scale factor of 0.7 is adopted to scale down the atomic charge in a non-polarisable force field during the molecular dynamics simulation. The other MD trajectory analysis and the binding energy calculation are described in "Supplementary Computational and Experimental Details" section.

\section{Experiments}

The materials and electrolyte preparation are described in the "Supplementary Computational and Experimental Details" section. The characterization methods of differential scanning calorimetry (DSC), ionic conductivity, transference number, and plating/stripping measurements are all given in the same section in the supplementary file.

\section{Declarations}

\section{Online content}

Any methods, additional references, Nature Research reporting summaries, source data, extended data, supplementary information, acknowledgements, peer review information; details of author contributions and competing interests; and statements of data and code availability are available at https://doi.org/xxxx.xxxx 


\section{Data Availability}

The data that support the findings of this study are available from the corresponding authors upon reasonable request.

\section{Acknowledgements}

F.C., M.F. acknowledge the Australian Research Council (ARC) for funding via the Australian Centre for Electromaterials Science, grant CE140100012. The simulation work was undertaken with the assistance of resources provided at the $\mathrm{NCI}$ National Facility systems at the Australian National University through the National Computational Merit Allocation Scheme supported by the Australian Government. X.W acknowledges the financial support of the Australia-India Strategic Research Fund (AISRF 48515).

\section{Author contributions}

F.C. X.W. and M.F. conceived the idea. F.C. directed the project and conducted computational work. X.W. conducted experiments. The results were discussed with M.F and M.A. All authors participated in manuscript preparation.

\section{Competing interests}

The authors declare no competing interests.

\section{Additional information}

Supplementary information is available for this paper at http://xxxxxxx.

Correspondence and requests for materials should be addressed to F.C. or M. F.

Reprints and permissions information is available online at www.nature.com/reprints.

\section{References}

1. Lopez, J., Mackanic, D.G., Cui, Y. \& Bao, Z. Designing polymers for advanced battery chemistries. Nature Reviews Materials 4, 312-330 (2019).

2. Zhao, Q., Stalin, S., Zhao, C.-Z. \& Archer, L.A. Designing solid-state electrolytes for safe, energy-dense batteries. Nature Reviews Materials 5, 229-252 (2020).

3. Li, J. et al. Polymers in Lithium-lon and Lithium Metal Batteries. Advanced Energy Materials n/a, 2003239 (2021).

4. Mauger, A., Armand, M., Julien, C.M. \& Zaghib, K. Challenges and issues facing lithium metal for solid-state rechargeable batteries. Journal of Power Sources 353, 333-342 (2017).

5. Dong, T. et al. A multifunctional polymer electrolyte enables ultra-long cycle-life in a high-voltage lithium metal battery. Energy \& Environmental Science 11, 1197-1203 (2018). 
6. Fenton, D.E., Parker, J.M. \& Wright, P.V. Complexes of alkali metal ions with poly(ethylene oxide). Polymer 14, 589 (1973).

7. Armand, M. The history of polymer electrolytes. Solid State Ionics 69, 309-319 (1994).

8. Zhang, H. et al. Lithium bis(fluorosulfonyl)imide/poly(ethylene oxide) polymer electrolyte. Electrochimica Acta 133, 529-538 (2014).

9. Rosenwinkel, M.P. \& Schönhoff, M. Lithium transference numbers in PEo/LiTFSa electrolytes determined by electrophoretic NMR. Journal of the Electrochemical Society 166, A1977-A1983 (2019).

10. Zhao, Y. et al. Solid Polymer Electrolytes with High Conductivity and Transference Number of Li lons for Li-Based Rechargeable Batteries. Advanced Science 8, 2003675 (2021).

11. Zhao, Y. et al. Design Strategies for Polymer Electrolytes with Ether and Carbonate Groups for SolidState Lithium Metal Batteries. Chemistry of Materials 32, 6811-6830 (2020).

12. Zhang, H. et al. Suppressed Mobility of Negative Charges in Polymer Electrolytes with an EtherFunctionalized Anion. Angewandte Chemie - International Edition 58, 12070-12075 (2019).

13. Tominaga, Y., Nanthana, V. \& Tohyama, D. lonic conduction in poly(ethylene carbonate)-based rubbery electrolytes including lithium salts. Polymer Journal 44, 1155-1158 (2012).

14. Sun, B., Mindemark, J., Edström, K. \& Brandell, D. Polycarbonate-based solid polymer electrolytes for Li-ion batteries. Solid State lonics 262, 738-742 (2014).

15. Miranda, D.F., Versek, C., Tuominen, M.T., Russell, T.P. \& Watkins, J.J. Cross-linked block copolymer/ionic liquid self-assembled blends for polymer gel electrolytes with high ionic conductivity and mechanical strength. Macromolecules 46, 9313-9323 (2013).

16. Ben youcef, H., Garcia-Calvo, O., Lago, N., Devaraj, S. \& Armand, M. Cross-Linked Solid Polymer Electrolyte for All-Solid-State Rechargeable Lithium Batteries. Electrochimica Acta 220, 587-594 (2016).

17. Bouchet, R. et al. Single-ion BAB triblock copolymers as highly efficient electrolytes for lithium-metal batteries. Nature Materials 12, 452-457 (2013).

18. Zhang, H. et al. Single lithium-ion conducting solid polymer electrolytes: advances and perspectives. Chemical Society Reviews 46, 797-815 (2017).

19. Li, J. et al. Synthesis of Sodium Poly[4-styrenesulfonyl(trifluoromethylsulfonyl)imide]-coethylacrylate] Solid Polymer Electrolytes. Electrochimica Acta 175, 232-239 (2015).

20. Girard, G.M.A. et al. Sustainable, Dendrite Free Lithium-Metal Electrode Cycling Achieved with Polymer Composite Electrolytes Based on a Poly(Ionic Liquid) Host. Batteries \& Supercaps 2, 229239 (2019).

21. Wang, X. et al. Poly(ionic liquid)s/Electrospun Nanofiber Composite Polymer Electrolytes for High Energy Density and Safe Li Metal Batteries. ACS Applied Energy Materials 2, 6237-6245 (2019).

22. Ohno, H., Yoshizawa, M. \& Ogihara, W. Development of new class of ion conductive polymers based on ionic liquids. Electrochimica Acta 50, 255-261 (2004). 
23. Zhang, S.-Y. et al. Poly(ionic liquid) composites. Chemical Society Reviews 49, 1726-1755 (2020).

24. Mecerreyes, D. Polymeric ionic liquids: Broadening the properties and applications of polyelectrolytes. Progress in Polymer Science 36, 1629-1648 (2011).

25. Safa, M., Chamaani, A., Chawla, N. \& El-Zahab, B. Polymeric Ionic Liquid Gel Electrolyte for Room Temperature Lithium Battery Applications. Electrochimica Acta 213, 587-593 (2016).

26. Wang, X. et al. Poly(lonic Liquid)s-in-Salt Electrolytes with Co-coordination-Assisted Lithium-lon Transport for Safe Batteries. Joule 3, 2687-2702 (2019).

27. Wang, X. et al. Toward High-Energy-Density Lithium Metal Batteries: Opportunities and Challenges for Solid Organic Electrolytes. Advanced Materials 32, 1905219 (2020).

28. Angell, C.A., Liu, C. \& Sanchez, E. Rubbery solid electrolytes with dominant cationic transport and high ambient conductivity. Nature 362, 137-139 (1993).

29. Xu, W., Wang, L.-M. \& Angell, C.A. "PolyMOB"-lithium salt complexes: from salt-in-polymer to polymer-in-salt electrolytes. Electrochimica Acta 48, 2037-2045 (2003).

30. Forsyth, M., Sun, J., Macfarlane, D.R. \& Hill, A.J. Compositional dependence of free volume in PAN/LiCF3SO3 polymer-in-salt electrolytes and the effect on ionic conductivity. Journal of Polymer Science, Part B: Polymer Physics 38, 341-350 (2000).

31. Zhang, Z., Nasrabadi, A.T., Aryal, D. \& Ganesan, V. Mechanisms of Ion Transport in Lithium SaltDoped Polymeric lonic Liquid Electrolytes. Macromolecules 53, 6995-7008 (2020).

32. Giffin, G.A. et al. Connection between Lithium Coordination and Lithium Diffusion in [Pyr1201] [FTFSI] Ionic Liquid Electrolytes. ChemSusChem 11, 1981-1989 (2018).

33. Kondou, S. et al. Ionic transport in highly concentrated lithium bis(fluorosulfonyl)amide electrolytes with keto ester solvents: structural implications for ion hopping conduction in liquid electrolytes. Physical Chemistry Chemical Physics 21, 5097-5105 (2019).

34. Suo, L. et al. "Water-in-salt" electrolyte enables high-voltage aqueous lithium-ion chemistries. Science 350, 938 (2015).

35. Yamada, Y. \& Yamada, A. Review-Superconcentrated Electrolytes for Lithium Batteries. Journal of the Electrochemical Society 162, A2406-A2423 (2015).

36. Chen, F., Howlett, P. \& Forsyth, M. Na-lon Solvation and High Transference Number in Superconcentrated Ionic Liquid Electrolytes: A Theoretical Approach. The Journal of Physical Chemistry C 122, 105-114 (2018).

37. Matsumoto, K., Okamoto, Y., Nohira, T. \& Hagiwara, R. Thermal and Transport Properties of $\mathrm{Na}$ [N(SO2F)2]-[N-Methyl-N-propylpyrrolidinium][N(SO2F)2] lonic Liquids for Na Secondary Batteries. The Journal of Physical Chemistry C 119, 7648-7655 (2015).

38. Gao, X., Wu, F., Mariani, A. \& Passerini, S. Concentrated lonic-Liquid-Based Electrolytes for HighVoltage Lithium Batteries with Improved Performance at Room Temperature. ChemSusChem 12, 4185-4193 (2019). 
39. Ma, Q. et al. A new $\mathrm{Na}$ [(FSO2)(n-C4F9SO2)N]-based polymer electrolyte for solid-state sodium batteries. Journal of Materials Chemistry A 5, 7738-7743 (2017).

40. Liu, L. et al. In Situ Formation of a Stable Interface in Solid-State Batteries. ACS Energy Letters 4, 1650-1657 (2019).

41. Smith, W. \& Forester, T.R. DL_POLY_2.0: A general-purpose parallel molecular dynamics simulation package. J. Mol. Graphics. 14, 136-141 (1996).

42. Martínez, L., Andrade, R., Birgin, E.G. \& Martínez, J.M. PACKMOL: A package for building initial configurations for molecular dynamics simulations. J. Comput. Chem. 30, 2157-2164 (2009).

43. Lopes, J.N.C. \& Padua, A.A.H. CL\&P: A generic and systematic force field for ionic liquids modeling. Theor Chem Acc 131 (2012).

44. Dodda, L.S., Cabeza de Vaca, I., Tirado-Rives, J. \& Jorgensen, W.L. LigParGen web server: an automatic OPLS-AA parameter generator for organic ligands. Nucleic Acids Research 45, W331W336 (2017).

\section{Figures}

a

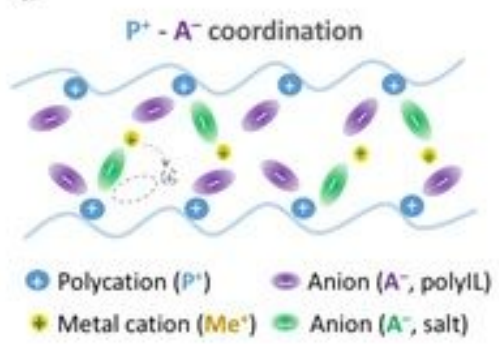

d

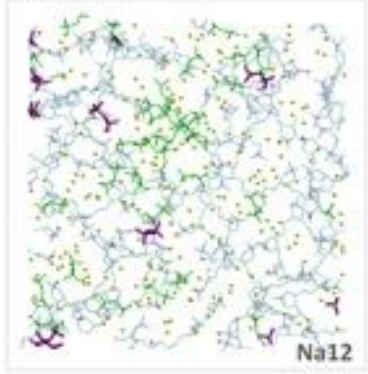

b $\mathrm{P}^{*}-\mathrm{A}^{-}-\mathrm{Me}^{*}$ co-coordination

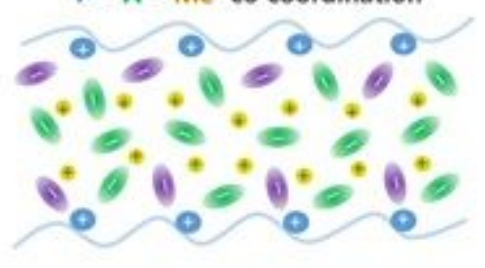

f

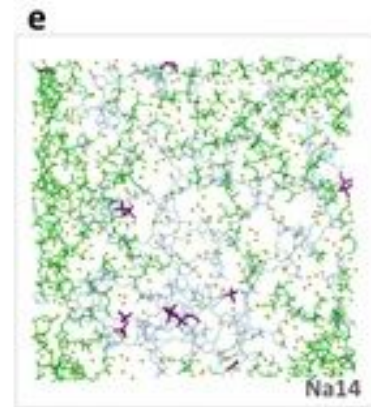

C
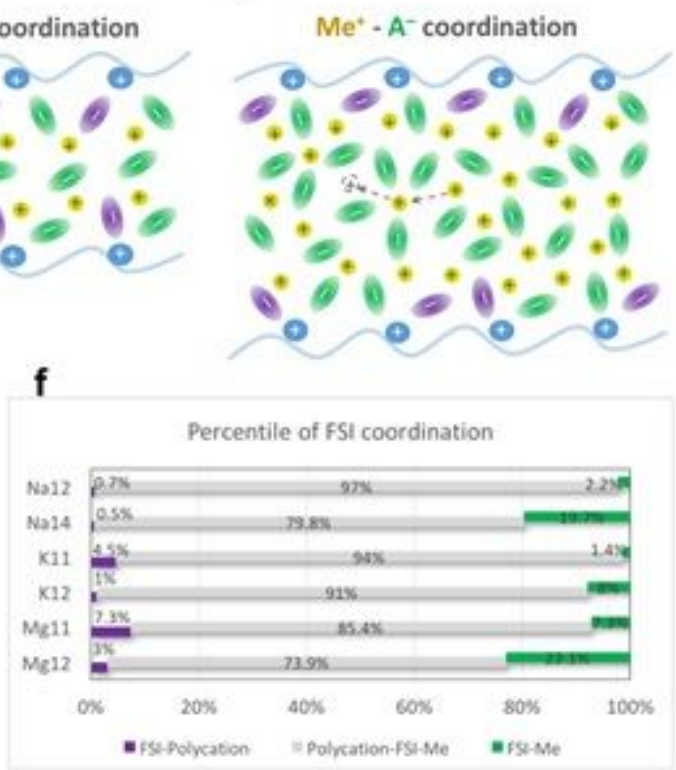

Figure 1

Cation-anion coordination in PolylLs. a-c, The schematic diagram to show the three dominant anion coordination states in PolylL with increasing salt concentration. The mechamisn of metal ion transport at low and high salt concentrations is illustrated. Color codes: polycation, blue; FSI- in PolyIL, Purple, FSIin salt, green; metal ion, yellow. d-e, Snapshots to show the equilibrium Na12 and Na14 systems. Only the $\mathrm{FSI}$ - in the P+-FSI- (purple) and Na+-FSI- (green) states are displayed. f, Percentile of FSI- in three coordination states for all investigated polylL systems. 

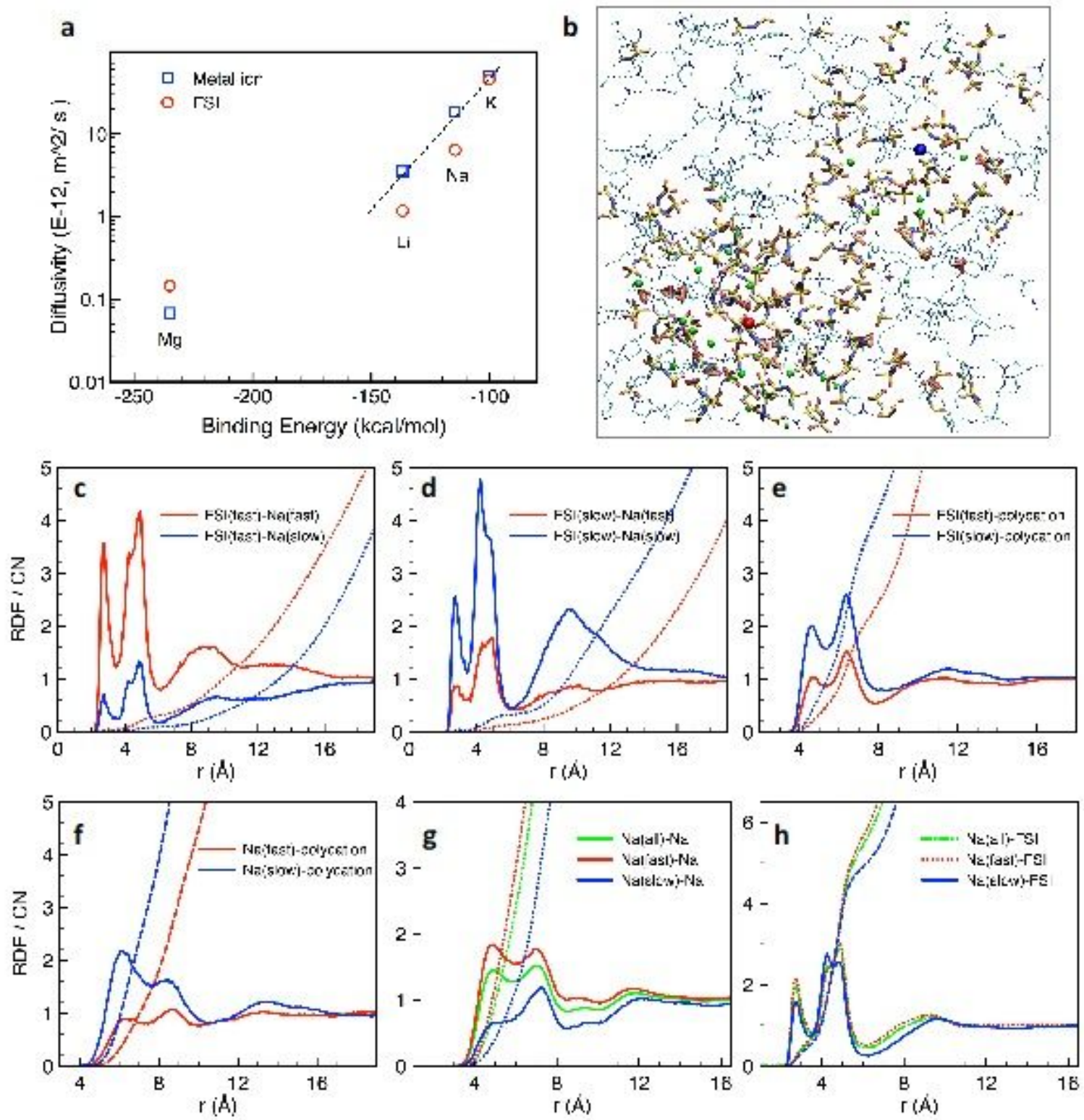

Figure 2

Diffusion of ions and analysis of the correlation between fast or slow ions. a, Log plot of diffusivity as a function of binding energy (Eb) of Me+-FSI- for K12, Na12, Mg11 and Li (1:1.5) systems. b, A snapshot showing fast (red) and slow (blue) $\mathrm{Na}+$ and their respective chemical environments. c-d RDF and CN of fast/slow FSI- with fast/slow Na+; e-f, RDF and CN of fast/slow FSI- or $\mathrm{Na}$ + with polycations; g-h, RDF and $\mathrm{CN}$ calculated between fast/slow and all $\mathrm{Na}+$ with $\mathrm{Na}+$ or FSI- . 
a
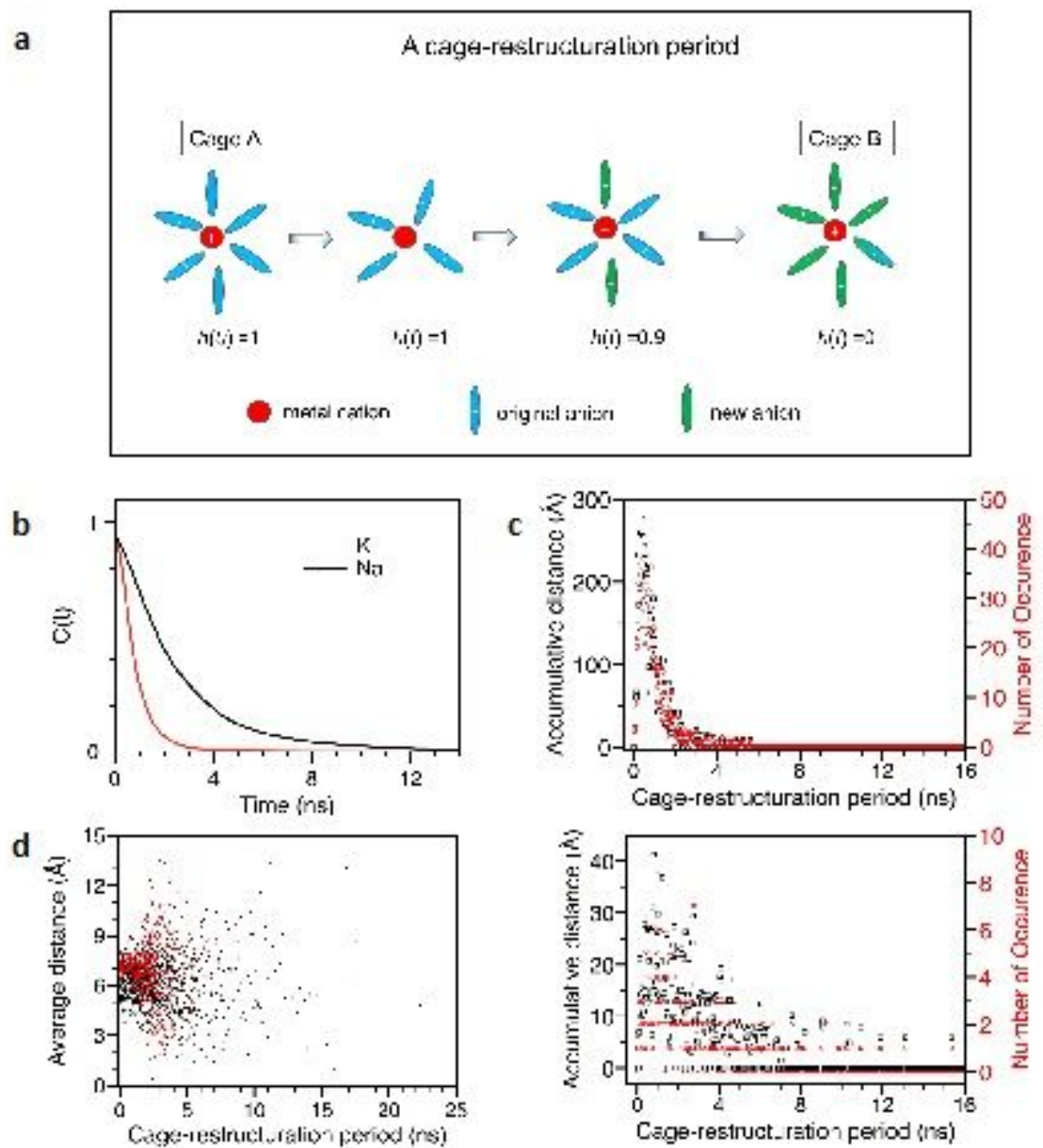

\section{Figure 3}

Analysis of metal ion cage restructuration and ion transport for the K12 and Na12 systems. a, Illustration of a cage-restructuration period and the corresponding coordination state function $\mathrm{h}(\mathrm{t})$. b, Cagerestructuration correlation function $c, e$, The number of occurrences of the same cage-restructuration period, and the accumulative final deviation distance of $\mathrm{Na}+$ and $\mathrm{K}+$, respectively. $\mathrm{d}$, The average distance $\mathrm{Na}+$ (black) and $\mathrm{K}+$ (red) moved in cage-restructuration periods of different lengths. 

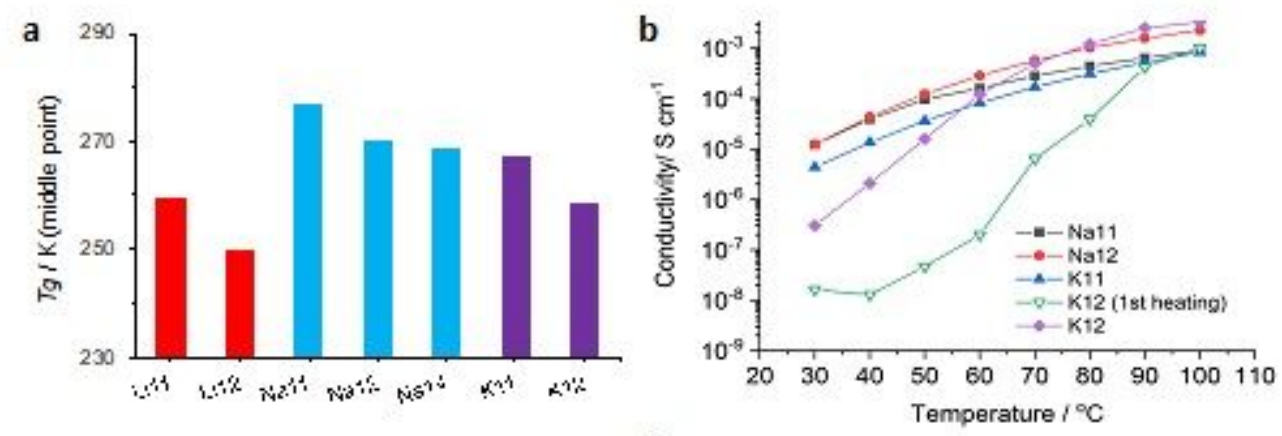

c

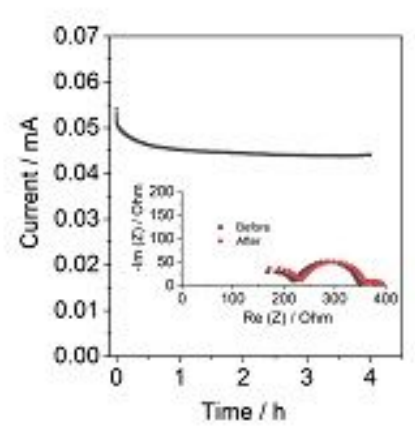

d

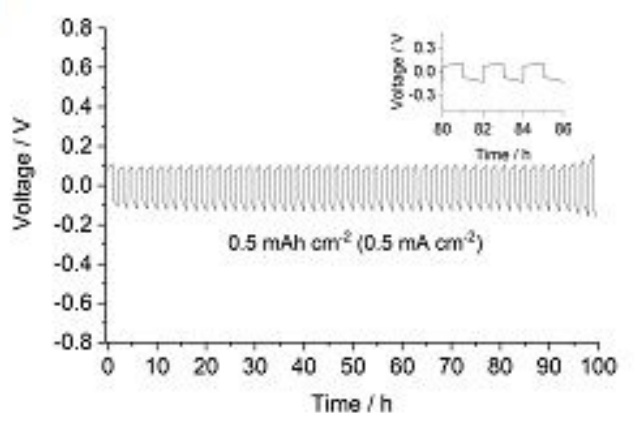

\section{Figure 4}

The thermal properties, ionic conductivities and electrochemical properties of PDADMA-based PEs. a, glass transition temperatures $(\mathrm{Tg})$ of PEs with various salt and concentrations. $b$, ionic conductivities as a function of temperature for $\mathrm{Na}$-, K- PolyIL PEs. c, the chronoamperometry profile of $\mathrm{Na} / \mathrm{Na} 12 \mathrm{PE} / \mathrm{Na}$ symmetric cell with a constant polarization voltage of $10 \mathrm{mV}$. Inset shows the Nyquist plots of the cell before and after polarization. $\mathrm{d}$, Na plating/stripping for a $\mathrm{Na} / \mathrm{Na} 12 \mathrm{PE} / \mathrm{Na}$ symmetric cell with an applied current density of $0.5 \mathrm{~mA} \mathrm{~cm}-2$. The electrochemical tests in c-d were performed at $80 \mathrm{oC}$.

\section{Supplementary Files}

This is a list of supplementary files associated with this preprint. Click to download.

- SupplementaryInformation.docx 